\title{
Obtenção de gás de síntese por reforma a seco do metano em unidade piloto
}

\author{
Obtaining syngas by dry reforming of \\ methane in a pilot unit
}

\author{
Lígia Gomes Oliveira ${ }^{1,2}$, Luana Pereira de Souza ${ }^{1,2}$, Bruna Machado ${ }^{1}$, \\ Sérgio Vitor Cavalcanti Trevisan ${ }^{1,2}$, Lázaro José Gasparrini ${ }^{1}$, Helton José Alves ${ }^{1,2}$
}

\footnotetext{
${ }^{1}$ Laboratório de Materiais e Energias Renováveis, LABMATER/UFPR, Universidade Federal do Paraná, Rua Pioneiro, n.2153, bloco IV, Jardim Dallas, CEP: 85.950-000, Palotina, Paraná, Brasil

${ }^{2}$ Programa de Pós-Graduação em Bioenergia, Universidade Federal do Paraná, Rua Pioneiro, n.2153, bloco IV, Jardim Dallas, CEP: 85.950-000, Palotina, Paraná, Brasil

e-mail: helquimica@gmail.com
}

\section{RESUMO}

A reforma a seco do metano (RS) é um processo catalítico endotérmico que ocorre entre 700 e $900{ }^{\circ} \mathrm{C}$, capaz de converter $\mathrm{CH}_{4}$ e $\mathrm{CO}_{2}$ (biogás) em $\mathrm{H}_{2}$ e $\mathrm{CO}$ (gás de síntese). Trabalhos têm sido elaborados visando o desenvolvimento de catalisadores ideais para a RS, buscando resolver os problemas encontrados, como é o caso da desativação do catalisador por formação de coque, que reduz o desempenho da produção do gás de síntese e pode obstruir o leito catalítico. Em trabalhos anteriores do Laboratório de Materiais e Energias Renováveis (LABMATER) da UFPR, foi desenvolvido o catalisador 20Ni/Si-MCM-41 que possui bom desempenho na conversão de $\mathrm{CH}_{4}$ e $\mathrm{CO}_{2}$ e baixa tendência à formação de coque $\left(\approx 10 \mathrm{mgC} \cdot \mathrm{gcat}^{-1} \cdot \mathrm{h}^{-1}\right)$, tratando-se de um material com carga nominal de $\mathrm{Ni}$ de $20 \%(\mathrm{~m} / \mathrm{m})$, suportado em uma peneira molecular a base de sílica mesoporosa (Si-MCM-41) de elevada área específica $\left(\approx 600 \mathrm{~m}^{2} \mathrm{~g}^{-1}\right)$. Entretanto, somente ensaios em unidades experimentais utilizando o catalisador em pó haviam sido realizados para testar a eficiência do material. Neste trabalho, foi utilizada uma unidade piloto contendo um reator contínuo de leito fixo, com o objetivo de avaliar a atividade do catalisador granulado e sua estabilidade na RS, de forma a subsidiar a construção futura de um reformador em escala industrial. Realizou-se quatro experimentos prévios com variação da composição reacional de 40-70\% $\mathrm{CH}_{4}: 30-60 \% \mathrm{CO}_{2}, 3$ g do catalisador $20 \mathrm{Ni} / \mathrm{Si}$ -

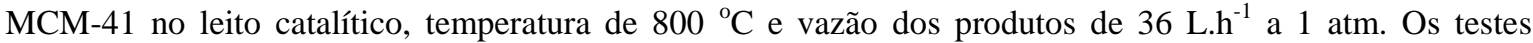
envolvendo variações nas composições de entrada com duração de 4 horas indicaram melhores resultados para a mistura $50 \% \mathrm{CH}_{4}: 50 \% \mathrm{CO}_{2}$. Também foi realizado um ensaio de 17 horas afim de verificar a estabilidade do catalisador, avaliar a formação de coque durante o tempo de reação e o desempenho da unidade piloto.

Palavras-chave: hidrogênio, sílica mesoporosa, Si-MCM-41.

\section{ABSTRACT}

Dry reforming of methane (DRM) is an endothermic catalytic process that occurs between 700 and $900{ }^{\circ} \mathrm{C}$, capable of converting $\mathrm{CH}_{4}$ and $\mathrm{CO}_{2}$ (biogas) into $\mathrm{H}_{2}$ and $\mathrm{CO}$ (syngas). Works have been undertaken in view of developing ideal catalyst for DRM, endeavoring to resolve the problems encountered, such as the case of catalyst deactivation due to coke formation, which reduces the performance of syngas production and may block the catalyst layer. In previous works by the Laboratory of Renewable and Materials Energies (LABMATER) at the UFPR, the catalyst 20Ni / Si-MCM-41 was developed, which has a good performance in the conversion of $\mathrm{CH}_{4}$ and $\mathrm{CO}_{2}$ and a low tendency to coke formation $\left(\approx 10 \mathrm{mgC} \cdot \mathrm{gcat}^{-1} \cdot \mathrm{h}^{-1}\right)$, being a material with a nominal Ni load of $20 \%(\mathrm{~m} / \mathrm{m})$, supported on a molecular sieve based on mesoporous silica (Si-MCM-41) with a high specific surface $\left(\approx 600 \mathrm{~m}^{2} \cdot \mathrm{g}^{-1}\right)$. However, only tests in experimental units using the catalyst in the powdered form have been performed to test the efficiency of the material. In this work, a pilot unit containing a continuous fixed bed reactor was used, with the objective of evaluating the activity of the granular catalyst and its stability in DRM, in order to subsidize the future construction of a reformer on an 
industrial scale. Four preliminary experiments were performed with a varying reaction composition of 40$70 \% \mathrm{CH}_{4}: 30-60 \% \mathrm{CO}_{2}, 3 \mathrm{~g}$ of the catalyst $20 \mathrm{Ni} / \mathrm{Si}-\mathrm{MCM}-41$ in the catalyst layer, temperature of $800{ }^{\circ} \mathrm{C}$ and a flow rate of the products of $36 \mathrm{Lh}^{-1}$ at $1 \mathrm{~atm}$. The tests involving variations in the entry compositions with a duration of 4 hours indicated better results for a mixture $50 \% \mathrm{CH}_{4}: 50 \% \mathrm{CO}_{2}$. Another test of 17 hours was performed, in order to verify the stability of the catalyst and evaluate coke formation during the reaction time and the performance of the pilot unit.

Keywords: hydrogen, mesoporous silica, Si-MCM-41.

\section{INTRODUÇÃO}

A reforma a seco do metano $\left(\mathrm{CH}_{4}\right)$ tem se mostrado uma alternativa para a geração de energia limpa, pois utiliza o $\mathrm{CH}_{4}$ e $\mathrm{CO}_{2}$, como matéria prima para obtenção do gás de síntese, que por sua vez é composto por $\mathrm{H}_{2}$ e CO (Equação 1), podendo ser empregado na produção de diversos compostos em refinarias e/ou no enriquecimento em $\mathrm{H}_{2}$ por meio de sua purificação. O gás de síntese pode ser obtido a partir de matérias primas ricas em hidrocarbonetos como carvão e gás natural, ou pela rota da biomassa, como é o caso do biogás [4,10], envolvendo principalmente os processos de gaseificação ou de reforma catalítica [5].

O processo de reforma do biogás ocorre sob pressão atmosférica em temperaturas entre 600 e $900{ }^{\circ} \mathrm{C}$, deste modo, a reação requer catalisadores seletivos e resistentes a agentes causadores de desativação e envenenamento, o que exige o desenvolvimento de catalisadores que sejam eficientes, estáveis e de baixo custo [4]. A obtenção do $\mathrm{H}_{2}$ via reforma do biogás, pode ser uma alternativa promissora para geração de energia limpa com elevada eficiência, principalmente, porque o hidrogênio renovável pode ser empregado em células a combustível, produzindo apenas água em sua combustão $[2,10]$.

$$
\mathrm{CH}_{4}+\mathrm{CO}_{2} \rightleftharpoons 2 \mathrm{H}_{2}+2 \mathrm{CO}\left(\Delta \mathrm{H}=+247 \mathrm{~kJ} \mathrm{~mol}^{-1}\right)
$$

Um desafio para a reforma a seco do metano reside no desempenho do catalisador, problema que está relacionado à desativação dos catalisadores pelo depósito de coque em sua superfície [14,15].

Diante o exposto, foi desenvolvido por AGUIAR et al [3], no Laboratório de Materiais e Energias Renováveis (LABMATER) da UFPR, Setor Palotina, um catalisador de Ni suportado com sílica mesoporosa do tipo MCM-41 que possui alta resistência e seletividade. O estudo variou a carga nominal de Ni em 10, 20 e $50 \%(\mathrm{~m} / \mathrm{m})$, para facilitar o entendimento, os catalisadores foram nomeados como Ni/Si-MCM-41. Para os testes reacionais foram adotadas as seguintes condições: granulometria do catalisador abaixo de $210 \mu \mathrm{m}$, composição de entrada de $50 \% \mathrm{CH}_{4}: 50 \% \mathrm{CO}_{2}$, velocidade espacial de $30 \mathrm{~L} \mathrm{~h}^{-1} \mathrm{~g}_{\text {cat }}{ }^{-1}$, temperatura de reação de $700{ }^{\circ} \mathrm{C}$. No leito reacional adicionou-se uma mistura homogeinizada de $0,3 \mathrm{~g}$ de catalisador e $2,25 \mathrm{~g}$ de sílica gel 60 para cromatografia, o tempo de reação foi de 4 horas. Os resultados obtidos de conversão foram de $35 \%$ de $\mathrm{CH}_{4}$ e $50 \%$ de $\mathrm{CO}_{2}$ para $10 \% \mathrm{Ni} / \mathrm{Si}-\mathrm{MCM}-41,93 \%$ de $\mathrm{CH}_{4}$ e $95 \%$ de $\mathrm{CO}_{2}$ para $20 \% \mathrm{Ni} / \mathrm{Si}-\mathrm{MCM}-41$ e $75 \%$ de $\mathrm{CH}_{4}$ e $70 \%$ de $\mathrm{CO}_{2}$ para $50 \% \mathrm{Ni} / \mathrm{Si}$-MCM-41. Ja os resultados de rendimento foram de $10 \%$ de $\mathrm{H}_{2} \mathrm{e}$ $20 \%$ de CO para $10 \% \mathrm{Ni} / \mathrm{Si}-\mathrm{MCM}-41,38 \%$ de $\mathrm{H}_{2}$ e $22,5 \%$ de CO para $20 \% \mathrm{Ni} / \mathrm{Si}-\mathrm{MCM}-41$ e $37 \%$ de $\mathrm{H}_{2}$ e $22,5 \%$ de CO [3]. Os autores constataram que o catalisador $20 \% \mathrm{Ni} / \mathrm{Si}-\mathrm{MCM}-41$ apresentou o melhor resultado.

Neste sentido, o catalisador $20 \% \mathrm{Ni} / \mathrm{Si}-\mathrm{MCM}-41$ foi selecionado e passou por um processo de adaptação para a reforma a seco em uma nova e maior unidade piloto, utilizando-o de forma granulada com diâmetro entre 355 e $500 \mu \mathrm{m}$ e $3,0 \mathrm{~g}$ de catalisador no leito reacional. Além do aumento na escala de 10 vezes em relação à massa do catalisador usada no reator, foi estudada a variação da composição dos gases de entrada $\left(\mathrm{CH}_{4} \mathrm{e} \mathrm{CO} \mathrm{CO}_{2}\right)$, sendo baseada na composição do biogás in natura, que consiste essencialmente em teores de $\mathrm{CH}_{4}$ de $40-70 \%$ e de $\mathrm{CO}_{2}$ de 30-60\%)[16].

\section{MATERIAIS E MÉTODOS}

Para o presente trabalho foram preparados catalisadores com teor metálico $20 \%$ de $\mathrm{Ni}$ impregnados ao suporte mesoporosos Si-MCM-41, estudado anteriormente por AGUIAR et al., [3], e posteriormente granulado com tamanhos dos grânulos variando entre $355-500 \mu \mathrm{m}$.

A granulação dos catalisadores é importante para dar resistência e evitar entupimento do leito. Para a granulação pesou-se $2 \mathrm{~g}$ de catalisador em pó e misturou-o a $2 \%$ de estearato de magnésio $\left(\mathrm{Mg}\left(\mathrm{C}_{18} \mathrm{H}_{35} \mathrm{O}_{2}\right)_{2}\right)$, material ligante, a mistura foi adicionada ao molde da prensa, o molde é feito de aço inox, cilíndrico com 9 mm de diâmetro interno, e na sequência, foi aplicado uma pressão de $159 \mathrm{MPa}$, obtendo assim pellets com dimensões de $9 \mathrm{~mm}$ de diâmetro por $2 \mathrm{~mm}$ de altura. Posteriormente os pellets foram calcinados em forno do tipo mufla com a rampa de $20^{\circ} \mathrm{C} / \mathrm{min}$ até $400{ }^{\circ} \mathrm{C}$, por 30 minutos. Após calcinação, desagregou-se em pistilo 
e os grânulos obtidos passou por seleção em peneiras com diâmetro entre 355 e $500 \mu \mathrm{m}$.

Após a obtenção dos catalisadores granulados, foram realizados os testes reacionais, experimentos prévios com variação da composição reacional de $40,50,60$ e $70 \%$ para o $\mathrm{CH}_{4}$ e $30,40,50$ e $60 \%$ para o $\mathrm{CO}_{2}, 3 \mathrm{~g}$ do catalisador $20 \mathrm{Ni} / \mathrm{Si}-\mathrm{MCM}-41$, temperatura de $800{ }^{\circ} \mathrm{C}$ e vazão dos produtos de $36 \mathrm{~L} \mathrm{~h}^{-1}$, sob pressão atmosférica.

Os ensaios reacionais foram realizados em uma unidade piloto (Figura 1b), disponível no LABMATER/UFPR. O esquema da unidade piloto (Figura 1a) está sendo representado da seguinte maneira : os gases (nitrogênio, hidrogênio, metano e dióxido de carbono) utilizados passam por controladores digitais de vazão e chegam no reator através de tubos de aço inox (8) e são conectados a um misturador de gases (12) através de engate rapido (11). Após a mistura, os gases $\mathrm{CH}_{4}$ e $\mathrm{CO}_{2}$ (ambos com pureza 99,99\% da White Martins) são aquecidos no forno de pré-aquecimento (13), e em seguida é aquecida no forno reator (21) e posterior segue para o reator tubular de aço inox 316 apresenta diâmetro externo de 1/2" (23) onde ocorre a reação de reação.

a)

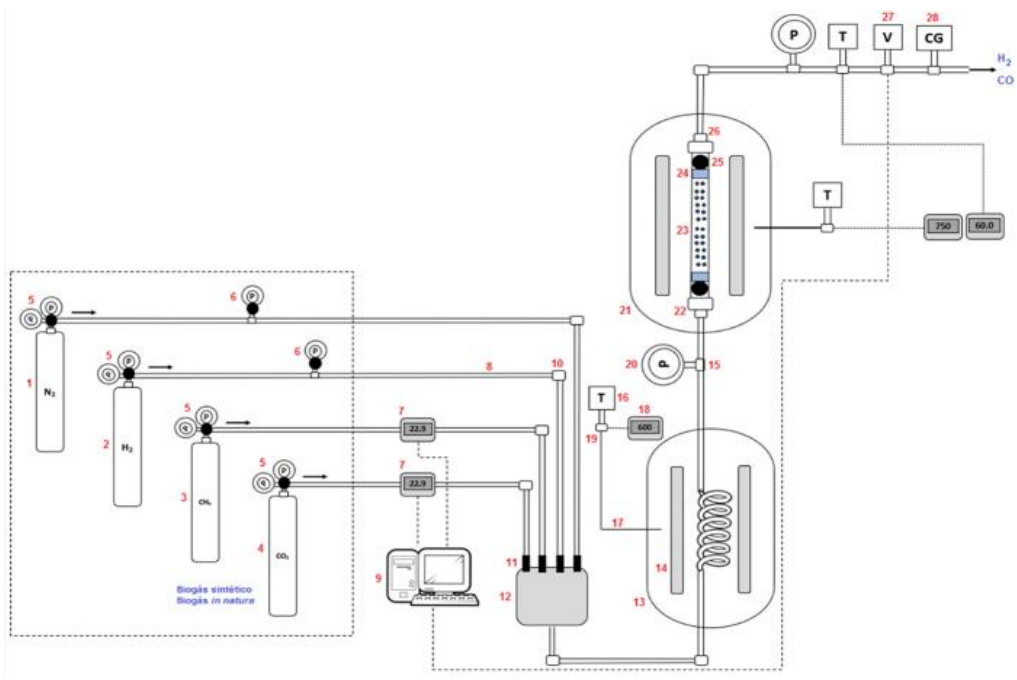

b)

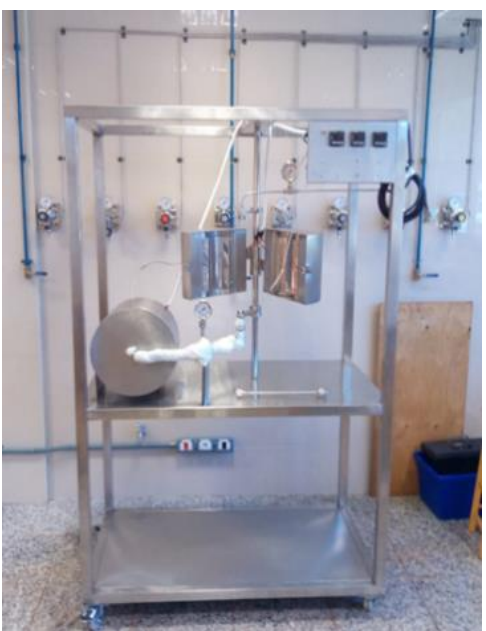

Figura 1: Esquema de unidade piloto para conversão do biogás em gás de síntese (a) e imagem da unidade piloto (b).

No reator, foram adicionados $3 \mathrm{~g}$ do catalisador granulado e posteriormente passou-se um fluxo de gás nitrogênio (gás inerte) por 30 minutos com uma vazão de $40 \mathrm{~m} \mathrm{~s}^{-1}$, para eliminar o oxigênio presente na linha.

Após purgar a linha com $\mathrm{N}_{2}$ (pureza > 99,999\%), iniciou-se o processo de ativação do catalisador, utilizando o $\mathrm{H}_{2}$ (pureza > 99,999\%) para redução do $\mathrm{NiO}$ para $\mathrm{Ni}$ metálico, mais uma vez foi inserido o fluxo de $\mathrm{N}_{2}$ para eliminar o gás, e por fim, a reação teve início.

As variações nas proporções de entrada foram nomeadas a seguir.

Tabela 1: Proporções de entrada de gases para cada ensaio reacional

\begin{tabular}{c|c|c}
\hline ENSAIO & $\mathrm{CH}_{4}(\%)$ & $\mathrm{CO}_{2}(\%)$ \\
\hline $\mathrm{R} 1$ & 40 & 60 \\
\hline $\mathrm{R} 2$ & 50 & 50 \\
\hline $\mathrm{R} 3$ & 60 & 40 \\
\hline $\mathrm{R} 4$ & 70 & 30 \\
\hline
\end{tabular}

Para estes ensaios foram avaliados conversão dos gases de entrada, fração molar, rendimento do $\mathrm{H}_{2} / \mathrm{CO}$ e a razão molar para cada condição reacional.

O tempo de reação foi de 4 horas, sendo as amostras coletadas a cada uma hora e analisadas por 
cromatografia gasosa. Os resultados do produto gasosos foram utilizados da quarta hora, pois é quando já existe uma estabilidade na reação.

O cromatógrafo utilizado para análise do produto gasoso foi o cromatógrafo à gás Agilent 7890A (colunas HP-PLOT/U e HP Mol. sieve $30 \mathrm{~m}$ x 0,530 mm x 20,00 $\mu \mathrm{m}$, split a $100 \mathrm{~mL} / \mathrm{min}, 3,9 \mathrm{~mL} / \mathrm{min}$ de Ar na fase móvel, volume de injeção na coluna de $0,01 \mu \mathrm{L}$, utilizando detector TCD), os gases de arraste usados no equipamento foram argônio, hidrogênio e ar sintético (pureza > 99,999\% da Air Liquide).

\subsection{Caracterização dos catalisadores}

Os catalisadores foram caracterizados antes e após os ensaios, pelas técnicas de microscopia eletrônica de varredura (MEV), difratometria de raios X (DRX) e análise termogravimétrica (ATG).

Para análise de MEV foi utilizado o microscópio eletrônico de varredura, FEI Quanta 440, da Central de Microscopia Eletrônica da UFPR - Setor Palotina. As amostras, em forma de pó granulado, foram espalhadas sobre o porta amostra de alumínio contendo uma fita de carbono dupla face, sendo posteriormente secas e metalizadas com uma fina camada de ouro na superfície (sputtering).

As análises de DRX, foram realizadas por em um Difratômetro Bruker, modelo D2-PHASER, utilizando as amostras na forma de pó e as análises realizadas no intervalo de $2 \theta$ entre $5^{\circ}$ e $70^{\circ}$, com radiação $\mathrm{Cu} \mathrm{K \alpha}(\lambda=1,5418 \AA)$, operando-se a $30 \mathrm{kV}, 10 \mathrm{~mA}$ e varredura contínua de $1,5^{\circ} \mathrm{min}^{-1}$.

As análises de ATG foram realizada com objetivo de determinar a perda de massa dos catalisadores coqueificados. A razão de aquecimento utilizada nos ensaios termogravimétricos foi de $10{ }^{\circ} \mathrm{C} \mathrm{min}{ }^{-1}$ até atingir $900^{\circ} \mathrm{C}$ e ar sintético (pureza > 99,999\%) como agente oxidante.

\section{RESULTADOS E DISCUSSÃO}

\subsection{Ensaios reacionais}

O catalisador $20 \% \mathrm{Ni}$ - Si-MCM-41 foi aplicado na reforma a seco do metano e os resultados de: conversão, rendimento e fração molar estão representados na Figura 2.

Os resultados de conversão (Figura 2a) indicam que para todas as condições de entrada a conversão foi superior a $88 \%$ para o $\mathrm{CH}_{4}$ e $85 \%$ para o $\mathrm{CO}_{2}$. Isso indica que mesmo com as variações nas composições de entrada dos gases, o catalisador possui boa eficiência no processo de conversão.

a)

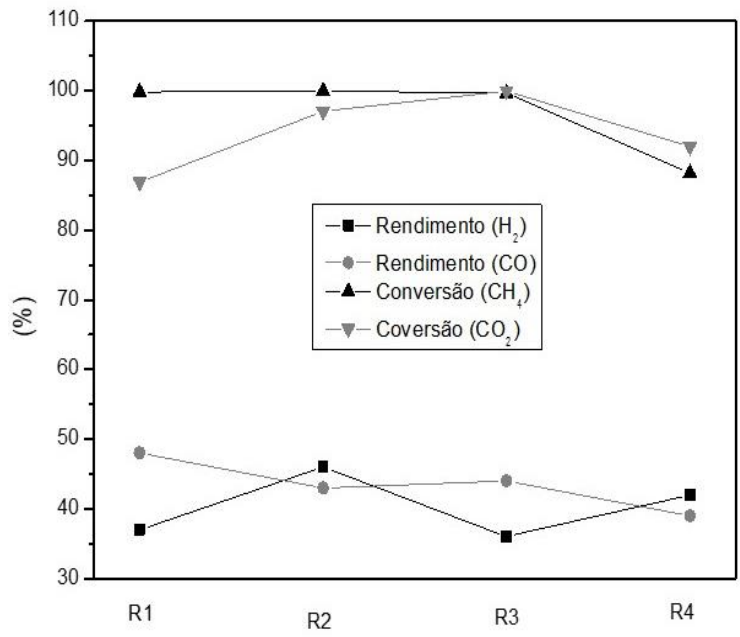

b)

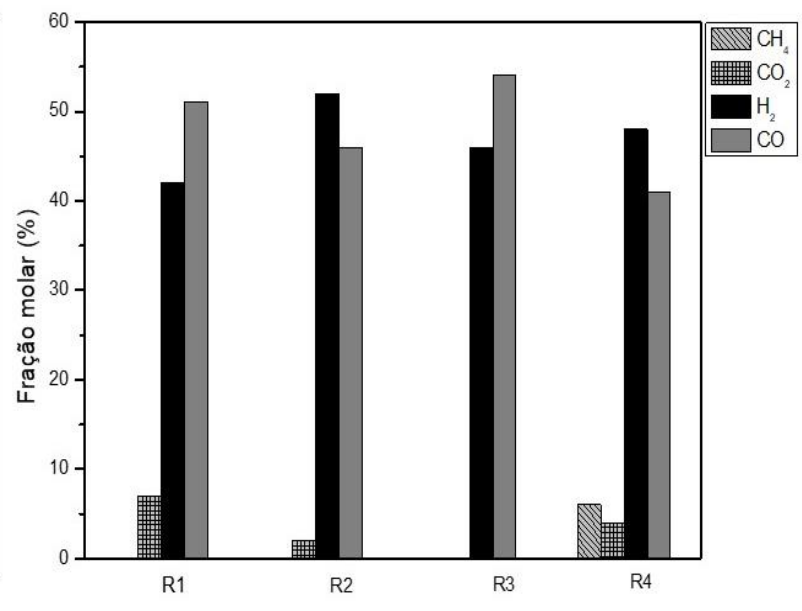

Figura 2: a) Conversão e rendimento e b) Fração molar dos gases obtidos nos testes catalíticos (4 horas) com o catalisador $20 \% \mathrm{Ni}-\mathrm{Si}-\mathrm{MCM}-41$

A fração molar dos gases de saída do reator está sendo representada na Figura 2b, para os quatro ensaios realizados, nota-se que apenas em R2 e R3 houve conversão próxima de $100 \%$ de $\mathrm{CH}_{4}$ e $\mathrm{CO}_{2}$, conforme confirmado na Figura 2a, os demais ensaios apontaram presença dos gases de entrada não convertidos, porém com valores abaixo de $6 \%$ para o $\mathrm{CH}_{4}$ e $7 \%$ para o $\mathrm{CO}_{2}$. 
Traçando um comparativo das condições reacionais é possível observar que o melhor resultado está sendo apontado em R2, pois possui conversão satisfatória, o rendimento dos gases (Figura 2a) possuem valores próximos $46 \%\left(\mathrm{H}_{2}\right)$ e $43 \%(\mathrm{CO})$ e fração molar de $52 \%$ e $46 \%$ para $\mathrm{H}_{2}$ e $\mathrm{CO}$, respectivamente. Nas demais condições as frações molares se afastam da estequiomentria desejada, quando as frações molares do $\mathrm{H}_{2}$ e CO se diferem significativamente isso pode indicar reações paralelas, como pode ser observado em R1, R3 e R4.

Dentre as reações paralelas que podem ocorrer no processo de reforma a seco do metano estão: deslocamento gás-água (water-gas shift), onde o $\mathrm{CO}$ reage com $\mathrm{H}_{2} \mathrm{O}$ para formar $\mathrm{CO}_{2}$ e $\mathrm{H}_{2}$, desta forma é possível observar maior fração molar do $\mathrm{H}_{2}$ frente ao $\mathrm{CO}$ e o deslocamento gás-água inversa que ocorre quando o $\mathrm{H}_{2}$ reage com o $\mathrm{CO}_{2}$ e forma $\mathrm{CO}$ e $\mathrm{H}_{2} \mathrm{O}[3,4]$. Neste sentido, é possível comprovar os bons resultados da condição reacional R2, pois seguem a estequimetria da reação.

\subsection{Reação de estabilidade (17 horas)}

Após as reações variando os catalisadores e proporções de entrada dos gases, foi escolhida uma condição reacional para avaliar a estabilidade do catalisador, o teste ocorreu com duração máxima de 17 horas e a condição reacional foi a R1, pois esta obteve o pior resultado como visto anteriormente. Esta escolha se justificou pelo fato de que é de interesse deste trabalho conhecer o desempenho do reator em condições adversas.

Como pode ser observado nas Figuras 3a e b, a reação permaneceu estável, sendo que a conversão dos gases de entrada foi de aproximandamente $100 \%$ para $\mathrm{CH}_{4}$ e $98 \%$ para o $\mathrm{CO}_{2}$. $\mathrm{O}$ redimento foi de aproximadamente $50 \%$ para $\mathrm{H}_{2}$ e $38 \%$ para o $\mathrm{CO}$, sendo favorável para a formação do $\mathrm{H}_{2}$. Na Figura 3a observa-se resultados conversão proximos de $100 \%$ para o $\mathrm{CH}_{4}$, assim como em $\mathrm{R} 1$, e $99 \%$ para o $\mathrm{CO}_{2}$, o que difere de R1(Figura 3a) que obteve conversão abaixo de $90 \%$.

A Figura $3 b$ aponta os resultados das frações molares dos gases na saída do reator, pode-se observar que não foi identificado o $\mathrm{CH}_{4}$ nos resultados e apenas uma pequena fração de $\mathrm{CO}_{2}$, sendo o rendimento obtido para o $\mathrm{H}_{2}$ de $50 \%$ (Figura 3a) e de $38 \%$ para o $\mathrm{CO}$. Com o baixo rendimento do $\mathrm{CO}$, mesmo com a conversão em quase $100 \%$ dos reagentes, o que pode indicar formação de $\mathrm{H}_{2} \mathrm{O}$ e/ou coque.

a)

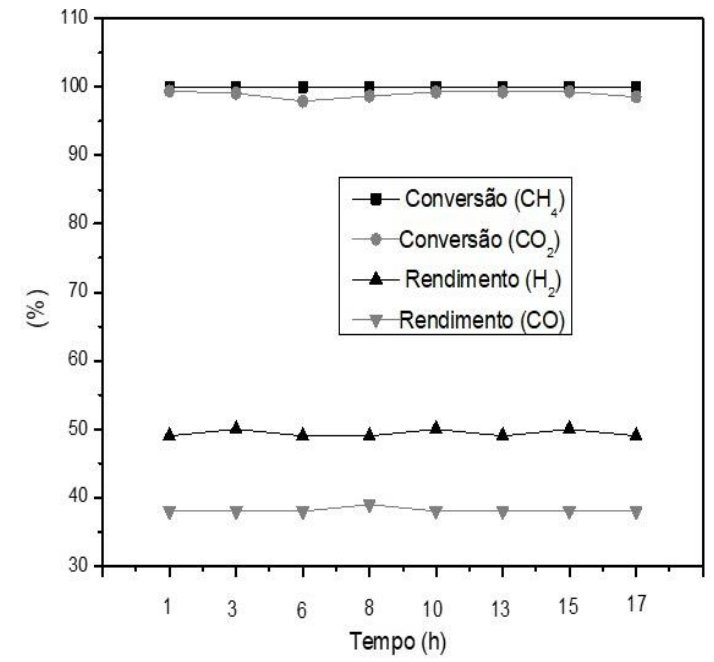

b)

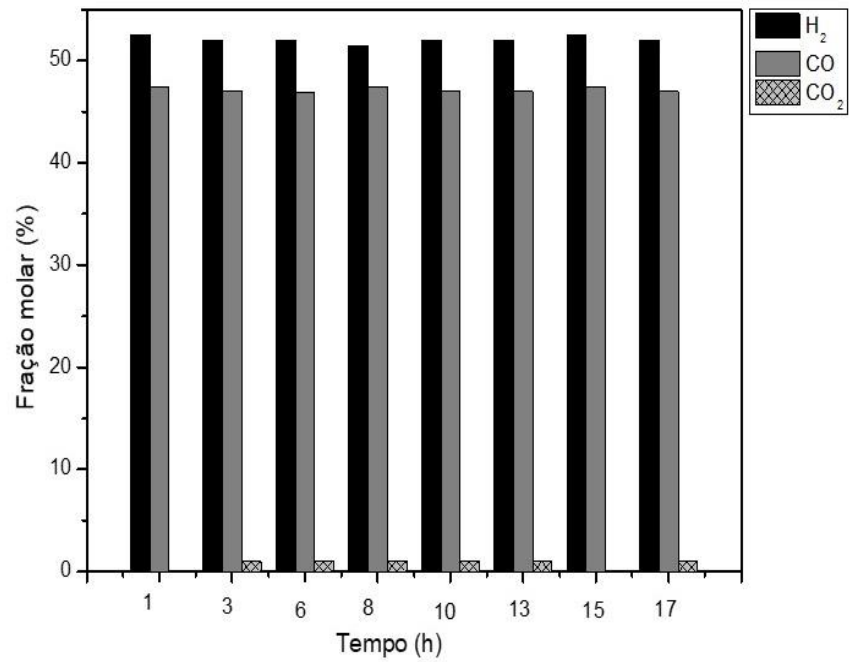

Figura 3: a) Conversão e rendimento e b) Fração molar dos gases obtidos nos testes catalíticos (17 horas) com o catalisador $20 \% \mathrm{Ni}-\mathrm{Si}-\mathrm{MCM}-41$.

Durante as 17 horas de reação não houveram oscilações expressivas nos resultados, o que ressalta o bom desempenho do catalisador e a estabilidade do reator. O trabalho realizado por AGUIAR et al.,[3], demonstrou que o catalisador 20\% Ni- Si-MCM-41 também obteve boa estabilidade catalítica.

\subsection{Caracterização dos catalisadores}

Os resultados de MEV são apresentados na Figura 4, onde mostra a morfologia dos catalisadores $20 \%$ de $\mathrm{Ni}$, 
antes, e após as reações de 4 e 17 horas, respectivamente. Os catalisadores apresentaram partículas esféricas, com a deposição de pequenas espécies sobre as superfícies destas partículas, provavelmente associados com a presença de óxido de níquel de acordo foram observados anteriormente por AGUIAR et al [2].

Na Figura 4b, pode-se observar semelhança com a Figura 4a, indicando que o material não sofreu degradação durante a reação, nem formação aparente de coque, porém, na Figura 4c é possível visualizar a aglomeração das partículas, o que pode indicar formação de coque.

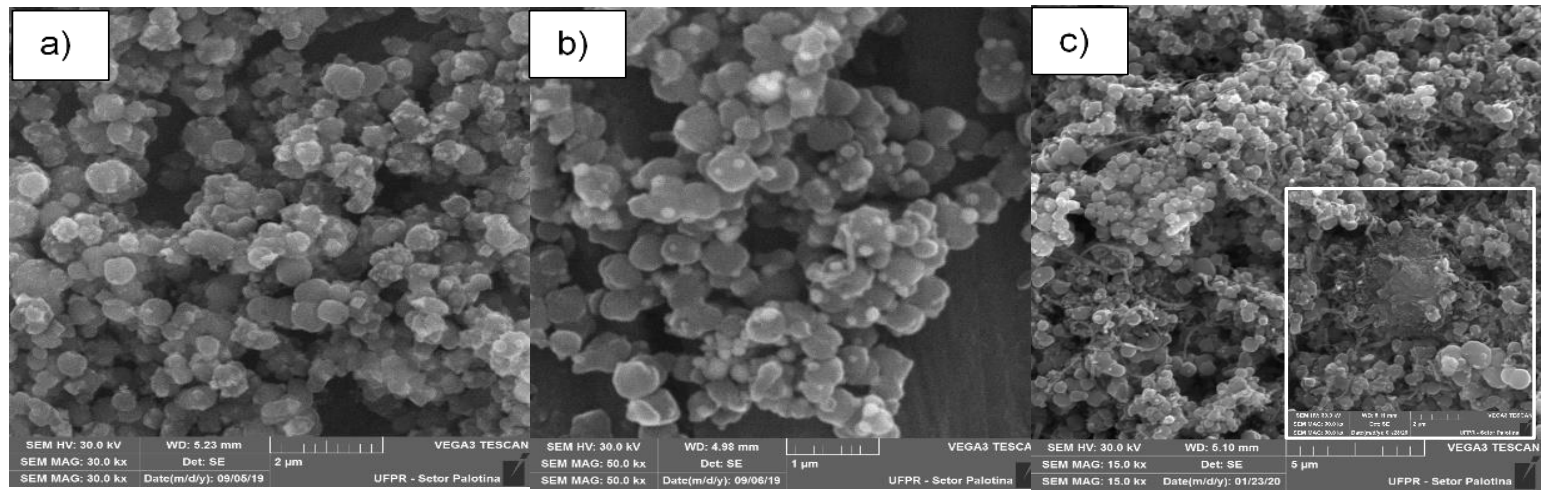

Figura 4: MEV dos catalisadores de 20\%Ni- Si-MCM-41: a) antes da reação; b) após as reações (4 horas) e c) após a reação $17 \mathrm{~h}$.

Os resultados das análises de DRX estão expostos na Figura 5.

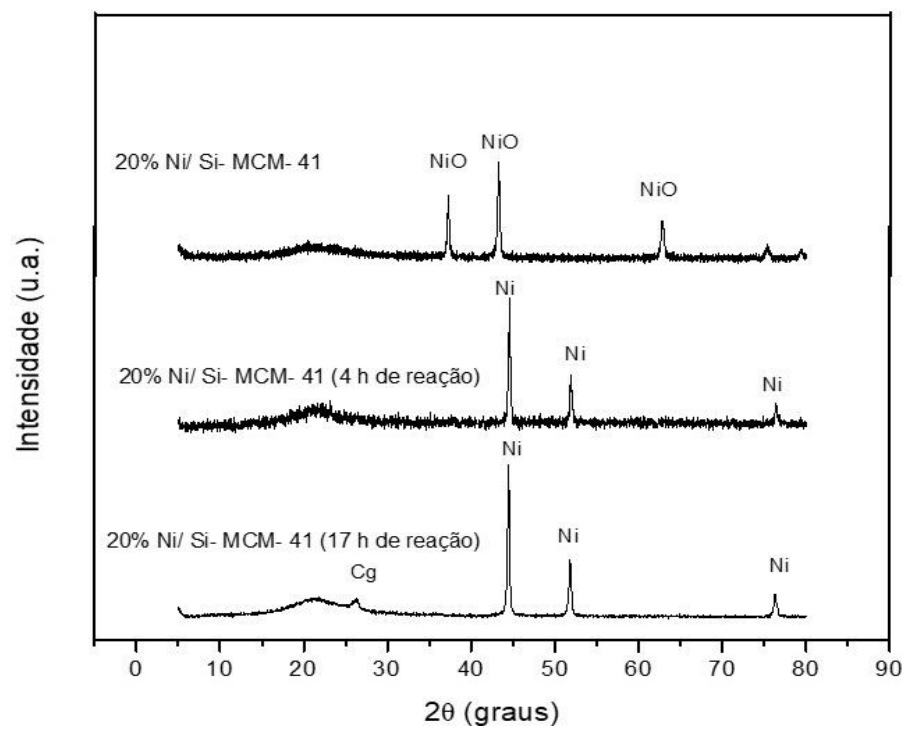

Figura 5: DRX do catalisador de 20\%Ni- Si-MCM-41 antes da reação, após 4 e 17 h de reação.

O difratograma (Figura 5) do catalisador ensaiado antes da reação revelou a presença de picos cristalinos em $37,28^{\circ}, 43,30^{\circ}$ e $62,92^{\circ}$, atribuídos ao $\mathrm{NiO}$ [6]. A análise realizada com o catalisador utilizado na reação de 4 horas indicou picos cristalinos localizados em $44,51^{\circ}, 51,85^{\circ}$ e $76,37^{\circ}$, associados ao $\mathrm{Ni}$ metálico [8], o que comprova a ausência de coque, porém para a reação de 17 horas, além dos picos associados ao $\mathrm{Ni}$, foi observado um pico em 26,59, o que indica formação de carbono grafite. A fase grafite deveria fornecer uma série de picos, sendo o mais intenso correspondente ao plano $002\left(2 \theta=26,6^{\circ}\right)$ [7], porém foi observado apenas o pico principal, o que comprova baixa formação de coque na reação de 17 horas.

A análise termogravimétrica (Figura 6), realizada em atmosfera oxidante (ar sintético), não registrou perda de massa para o catalisador após a reação de 4 horas, indicando que não havia coque, porém houve um aumento de massa até 700 graus, devido o Ni passar por um processo de redução in situ e a análise ter sido realizada em atmosfera oxidante, acredita-se que ocorreu a oxidação do níquel metálico (Ni) para óxido de níquel (NiO). No resultado da reação de 17 horas é visível a perda de massa de $7 \%$ entre 500 e 600 graus, visto que a formação de carbono foi de $4,42 \times 10^{-3} \mathrm{~g}_{\text {carbono }} \mathrm{g}_{\text {catalisador }}{ }^{-1} \mathrm{~h}^{-1}$. 


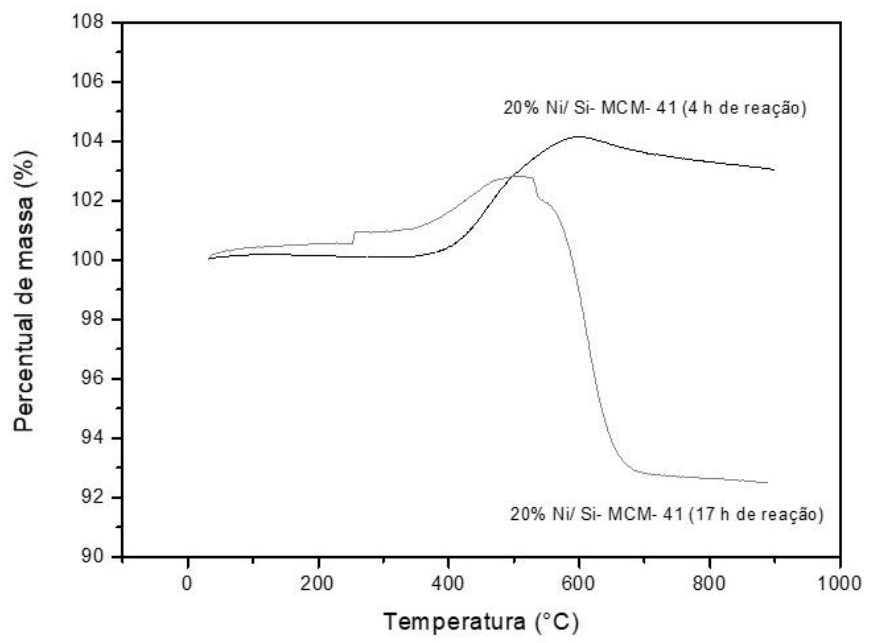

Figura 6: ATG do catalisador 20\%Ni- Si-MCM-41 após as reações 4 e 17 horas.

\section{CONCLUSÕES}

A variação de composição de mistura gasosa na alimentação da unidade piloto, revelou que a faixa ideal para obtenção seletiva de $\mathrm{H}_{2}$ (48\% a 50\%) e CO (49\% a 51\%), com conversões dos reagentes acima de $97 \%$, está entre 50 e $60 \%$ de $\mathrm{CH}_{4}$ e 40 e $50 \%$ de $\mathrm{CO}_{2}$. A análise de ATG em atmosfera oxidante (ar sintético) não registrou perda de massa para o catalisador após $4 \mathrm{~h}$ de reação, indicando ausência de coque. Porém, após 17 $\mathrm{h}$ de reação foi sendo observada perda de massa de $7 \%$ atribuída à formação de coque, mas a formação de coque é relativamente baixa, fazendo um comparativo com outros trabalhaos de pesquisa do grupo LABMATER, de BACH [17] que utilizou catalisador com $10 \%$ de teor de Ni suportado com Alumina e promovido com 5\% de Mg e SCHAFFNER [18] que utilizou catalisador com 10\% de teor de Ni suportado com Alumina sem promotor obtiveram perdas de massa de $10,12 \%$ e $8,33 \%$, respectivamente, para os testes feitos com apenas 4 horas de reação. O coque apresentado no teste de estabilidade pode ser confirmado nas micrografias de MEV e por DRX devido à presença de um pico cristalino atribuído ao carbono. A DRX dos catalisadores ensaiados antes da reação revelou a presença de picos cristalinos associados ao óxido de níquel, e após as reações de 4 e $17 \mathrm{~h}$, confirmou a presença de picos atribuídos à fase ativa do catalisador, ou seja, níquel metálico obtido pela ativação in situ do catalisador com $\mathrm{H}_{2}$ no leito catalítico, não sendo observadas alterações ocasionadas pela sinterização ou mudanças estruturais da fase ativa.

\section{AGRADECIMENTOS}

Agradecimentos à Companhia Paranaense de Energia - Copel. O projeto é parte do P\&D ANEEL (PD 28660470/2017)

\section{BIBLIOGRAFIA}

[1] ABDIN, Z., ZAFARANLOO, A., RAFIEE, A., et al., "Hydrogen as an energy vector", Renewable and Sustainable Energy Reviews, v. 120, pp. 109620, 2020.

[2] ABE, J.O., AJENIFUJA, E., POPOOLA., O.M."Hydrogen energy, economy and storage: review and recommendation." International Journal of Hydrogen Energy, v.44, pp. 15072-15086 (2019).

[3] AGUIAR, M., CAZULA, B.B., COLPINI, L.M.S., et al., "Si-MCM-41 obtained from different sources of silica and its application as support for nickel catalysts used in dry reforming of methane", International Journal of Hydrogen Energy, v. 44, n. 60, pp. 32003-32018, 2019.

[4] ALVES, H.J., JUNIOR, C.B., NICKLEVICZ, R.R., et al., "Overview of hydrogen production technologies from biogas and the applications in fuel cells", International Journal of Hydrogen Energy, v. 38, n. 13, pp. 5215-5225, 2013.

[5] ANDERSSON, R., "Catalytic conversion of syngas to higher alcohols over MoS2-based catalysts". Tese de D.Sc., KTH Royal Institute of Technology, Stockholm, Sweden, 2015. 
[6] CAI, W., ZHANG, L.Y., REN, Y., et al., "Highly dispersed nickelcontaining mesoporous silica with superior stability in carbon dioxide reforming of methane: the effect of anchoring", Materials, v. 7, n. 3, pp. 2340-2355, 2014.

[7] CARUSO, F., JABLONSKI, E.L., GRAU, J. M., et al.“Crystallinityof coke on platinum-rhenium/alumina reforming catalyst during the commercial cycle", Applied catalysis, v. 51, n. 1, pp. 195-202, 1989.

[8] CORREIA, G.B., "Síntese e caracterização de nanopartículas de níquel para óptica não linear”. Tese de M.Sc., UFPE, Recife, PE, Brasil, 2008.

[9] FUKUROZAKI, S.H., “Avaliação do ciclo de vida de potenciais rotas de produção de hidrogênio: estudo dos sistemas de gaseificação da biomassa e de energia solar fotovoltaica”. Tese de D.Sc., USP, São Paulo, SP, 2011.

[10] RATHOD, V., BHALE, P.V., "Experimental investigation on biogas reforming for syngas production over an alumina based nickel catalyst", Energy Procedia, v. 54, pp. 236-245, 2014

[11] SALKUYEH, Y.K., II, T.A.A., "Combining coal gasification, natural gas reforming and external carbonless heat for efficient production of gasoline and diesel with $\mathrm{CO}_{2}$ capture and sequestration", Energy Conversion and Management, v. 74, pp. 492-504, 2013.

[12] SANTOS, F.M., SANTOS, F., “Combustível 'hidrogénio””. Millenium, pp. 252-270, 2005.

[13] VARGAS, R.A. et al., "Hidrogênio: o vetor energético do futuro", Centro de Ciência e Tecnologia de Materiais (CCTM), Instituto de Pesquisas Energéticas e Nucleares (IPEN), São Paulo, 2006.

[14] ZHANG, R.J., Xi A., G.F., et al., "Effect of support on the performance of Ni-based catalyst in methane dry reforming". Jornal de Combustivel Química e Tecnologia, v. 43, n. 11, pp. 1359-1365, 2015.

[15] SCHWENGBER, C.A., SILVA, F.A., SCHAFFNER, R.A., et al., "Methane dry reforming using $\mathrm{Ni} / \mathrm{Al}_{2} \mathrm{O}_{3}$ catalysts: Evaluation of the effects of temperature, space velocity and reaction time", Journal of Environmental Chemical Engineering, v. 4, n. 3, pp. 3688-3695, 2016.

[16] RYCKEBOSCH, E., DROUILLON, M., VERVAEREN, H., “Techniques for transformation of biogas to biomethane", Biomass And Bioenergy, v. 35, n. 5, pp.1633- 1645, 2011.

[17] BACH, V.R., "Efeito da adição de Mg como promotor em catalisadores Ni/AI2O3 aplicados à reforma a seco do metano", Tese de M.Sc., UFPR, Palotina, PR, 2016.

[18] SCHAFFNER, R.D.A. "Catalisadores $\mathrm{Ni} / \mathrm{Al}_{2} \mathrm{O}_{3}$ para a reforma a seco do metano: efeito da temperatura de calcinação do suporte e uso de promotor alcalino”, Tese de M.Sc., UFPR, Palotina, PR, 2018.

\section{ORCID}

Lígia Gomes Oliveira

Luana Pereira de Souza

Bruna Machado

Lázaro José Gasparrini

Sérgio Vitor C. Trevisan

Helton José Alves https://orcid.org/0000-0002-0851-1302

https://orcid.org/0000-0002-6119-2494

https://orcid.org/0000-0003-1305-6671

https://orcid.org/0000-0002-0015-5489

https://orcid.org/0000-0002-5605-1363

https://orcid.org/0000-0001-6942-1020 